\title{
Aplicativo móvel para mães sobre manejo de crianças em pós- operatório de ortopedia
}

\author{
Mobile app for mothers about managing children in the postoperative period of orthopedics \\ Aplicación móvil para madres sobre el manejo de niños en el postoperatorio de ortopedia \\ Benedito do Carmo Gomes Cantão ${ }^{1 *}$, Mariseth Carvalho de Andrade ${ }^{1}$, Anderson Bentes de Lima1.
}

\section{RESUMO}

Objetivo: Apresentar o processo de desenvolvimento e validação de um aplicativo para auxiliar as mães no cuidado de crianças no pós-operatório de ortopedia. Métodos: Trata-se de um estudo metodológico, conduzido com 8 enfermeiros e 11 mães. O processo compreendeu avaliação de funcionamento, interação do usuário, rapidez e qualidade como critérios para julgamento da usabilidade do aplicativo. A partir da escala de Likert, as variáveis foram analisadas por meio o coeficiente de Alfa de Cronbach e a concordância das respostas dos participantes pelo Índice de Validade de Conteúdo (IVC). Resultados: Dentre as respostas, dez itens obtiveram bom desempenho, com escore de IVC variando de 0,84 a 1,00, destaque para o item 6, que apresentou pontuação máxima; e apenas, o item 3 apresentou IVC abaixo do recomendado pela literature, que é em torno de 0,80 . Na avaliação da confiabilidade do questionário, obteve-se a Alfa de Cronbach de 0.906, resultado classificado como "Muito Alto", demostrando que os itens que compuseram o questionário são homogêneos. Conclusão: O grau de confiabilidade e concordância usados para validação do aplicativo, demostraram que o produto foi considerado válido, com taxa de aprovação maior que $86 \%$ nos dois índices de validação.

Palavras-chave: Tecnologia educacional, Aplicativos móveis, Ortopedia, Pós-operatório, Pediatria.

\begin{abstract}
Objective: To present the process of development and validation of an application to assist mothers in the care of children in the postoperative period of orthopedics. Methods: This is a methodological study, conducted with 8 nurses and 11 mothers. The process included evaluation of functioning, user interaction, speed and quality as criteria for judging the usability of the application. From the Likert scale, the variables were analyzed using the Cronbach's alpha coefficient and the agreement of the participants' responses by the Content Validity Index (CVI). Results: Among the responses, ten items performed well, with CVI score ranging from 0.84 to 1.00 , highlighting item 6 , which had the highest score; and only, item 3 presented CVI below that recommended by the literature, which is around 0.80 . In assessing the reliability of the questionnaire, Cronbach's alpha of 0.906 was obtained, a result classified as "Very High", showing that the items that made up the questionnaire are homogeneous. Conclusion: The degree of reliability and agreement used to validate the application, demonstrated that the product was considered valid, with an approval rate greater than $86 \%$ in both validation indexes.
\end{abstract}

Key words: Educational technology, Mobile apps, Orthopedics, Postoperative, Pediatrics.

\section{RESUMEN}

Objetivo: Presentar el proceso de desarrollo y validación de una aplicación para asistir a madres en el cuidado de niños en el postoperatorio de ortopedia. Métodos: Se trata de un estudio metodológico, realizado con 8 enfermeras y 11 madres. El proceso incluyó la evaluación del funcionamiento, la interacción del usuario, la velocidad y la calidad como criterios para juzgar la usabilidad de la aplicación. A partir de la escala Likert, las variables se analizaron mediante el coeficiente alfa de Cronbach y la concordancia de las respuestas de los participantes por el Índice de Validez de Contenido (IVC). Resultados: Entre las respuestas, diez ítems obtuvieron un buen desempeño, con puntaje CVI que varió de 0.84 a 1.00, destacando el ítem 6 , que tuvo el puntaje más alto; y solo, el ítem 3 presentó el CVI por debajo de lo recomendado por la literatura, que es alrededor de 0,80 . Al evaluar la confiabilidad del cuestionario se obtuvo un alfa de Cronbach de 0.906, resultado clasificado como "Muy Alto", mostrando que los ítems que componen el cuestionario son homogéneos. Conclusión: El grado de confiabilidad y concordancia utilizado para validar la aplicación, demostró que el producto se consideró válido, con una tasa de aprobación superior al $86 \%$ en ambos índices de validación.

Palabras clave: Tecnología educativa, Aplicaciones móviles, Ortopedia, Posoperatorio, Pediatría.

${ }^{1}$ Universidade do Estado do Pará (UEPA), Belém - PA. *E-mail: bcgcantao@hotmail.com SUBMETIDO EM: 3/2021 


\section{INTRODUÇÃO}

O conceito de trauma dar-se-á em uma lesão que varia de acordo com a sua gravidade, podendo ser produzida de forma intencional ou acidental por causas diversas, tais como: quedas, colisões, queimaduras elétricas, química, maus tratos, violência, etc., e que em curto período de tempo é capaz de acarretar diferentes distúrbios locais e sistêmicos do organismo humano, que incluem danos físicos e psicológicos e que em alguns casos podem levar a morte. Tais incidentes podem ocorrer no ambiente doméstico ou social (FILÓCOMO FRF, et al., 2017).

Os acidentes ou traumas ocorridos na infância no ambiente doméstico ou social nos últimos anos têm sido considerados como um desafio para a saúde pública, pois a nível mundial, ocupa a posição número um no ranking de causas de morte na infância, bem como elevado índice de implicações decorrentes das lesões. Segundo o Fundo das Nações Unidas para a Infância, presume-se que no mundo ocorra cerca de 875 mil óbitos anualmente oriundos de acidentes não intencionais e intencionais, o que representa cerca de 2.397 mortes por dia (BARCELOS RS, et al., 2017).

No Brasil, um levantamento realizado com 48.478 pessoas de todas as idades pelo Ministério da Saúde, demostrou que a violência e os acidentes são considerados como primeira causa de morte entre indivíduos 0 a 19 anos de idade, correspondendo a 30,67\% dos casos investigados. Em 2017 foram registrados no serviço de pronto socorro das capitais brasileiras, 44.204 mil atendimentos, sendo que $7.017(15,4 \%)$ envolvendo acidentes e quedas em crianças de 0 a 9 anos de idade (BRASIL, 2019).

Os traumas acabam por resultar em procedimentos cirúrgicos, os quais constituem uma inquietação com reflexos para o setor de saúde e paciente. Para os gestores, tal inquietação se refere ao impacto social e econômico, em especial sobre o Sistema único de Saúde (SUS). No caso dos pacientes e familiares se reflete no surgimento de diversos sentimentos como medos, ansiedades e dúvidas que repercutem no período de pós-operatório (BRASIL, 2019; SILVA JP e GARANHANI ML, 2011).

No que se refere ao impacto econômico, estima-se que o Brasil, em 2017, registrou um gasto de 1,4 bilhão de reais com internações para atendimentos de usuários nos serviços de urgência decorrente de causas externas (BRASIL, 2019). Por conseguinte, em virtude de os traumas conseguirem incapacitar o paciente para desenvolver suas próprias tarefas, bem como, o pós-operatório ser, consideravelmente, demorado, são necessários cuidados específicos durante o período pós cirurgia ortopédica, a saber: auxílio para mudança de posição, imobilização do membro afetado, curativos, alivio da dor, cuidados para evitar a infecção e a obstrução de um vaso sanguíneo, visto que qualquer procedimento cirúrgico acaba manifestando no paciente uma sequência de reações fisiológicas e psicológicas. Assim como, uso de amparo durante a deambulação e manutenção da autonomia (CUNHA MLR, et al., 2018; HERDMAN T, 2015; BUTCHER HK, et al., 2018).

É possível observar que no cotidiano do ambiente hospitalar, ainda é comum a modalidade verbal, como opção para orientações de pacientes de pós-operatório, e que frequentemente as informações são transmitidas apressadamente e com baixa assimilação do conteúdo, levando as mães a terem uma percepção equivocada do estado de saúde que aliado ao medo acaba gerando dúvidas, ansiedade e baixa a adesão ao plano terapêutico (SILVA JP e GARANHANI ML, 2011).

Com base no relatório de uma pesquisa realizada em 2018 pela Comissão de Saúde Global de Alta Qualidade, observou que os cuidados inapropriados e a fraca resolutividade do serviço de saúde estão relacionados a vários fatores tais como: consultas rápidas, falta de continuidade do cuidado e da comunicação que acabam gerando resultados adversos e sofrimentos desnecessários (KRUK ME, et al., 2018).

Vale ressaltar, que o uso do aplicativo como estratégia para o processo de aprendizagem das mães quanto ao manejo correto no pós-operatório de ortopedia, representa uma alternativa viável devido ao grande potencial de alcance. Visto que, nos últimos anos, vem ocorrendo o uso crescente de smartphones entre os brasileiros. De acordo com a pesquisa nacional feita pelo Instituto Brasileiro de Estatística, em 2018, estimava que aproximadamente $99,2 \%$ das pessoas acessam a internet por meio do celular, sendo que a principal atividade apontada por $95,5 \%$ das pessoas que usam a internet é troca de mensagens, áudio e imagens (IBGE, 2020). 
Assim sendo, a pesquisa teve como objetivo apresentar o processo de desenvolvimento e validação de um aplicativo para auxiliar as mães no cuidado de crianças no pós-operatório de ortopedia.

\section{MÉTODOS}

Trata-se de um estudo metodológico, desenvolvido na clínica pediátrica de um hospital público do interior do estado do Pará. A pesquisa obedeceu aos devidos preceitos éticos, portanto, seu início aconteceu após o aceite do Comitê de Ética em Pesquisa da Universidade do Estado do Pará (UEPA) pelo parecer no 3.350.668, CAAE: 12048719.2.0000.5170. Ainda, todos os participantes assinaram o Termo de Consentimento Livre e Esclarecido (TCLE). O desenvolvimento e validação do aplicativo aconteceu em cinco etapas.

A primeira etapa consistiu na realização de um estudo quantitativo epidemiológico, de caráter descritivo e corte transversal retrospectivo, utilizando-se o período de 2017 a 2018, para traçar o perfil epidemiológico de cirurgias ortopédicas pediátricas do hospital em questão (CANTÃO BCG, et al., 2021). Esta etapa teve como intuito realizar o levantamento dos principais tipos de traumas ortopédicos pediátricos da instituição, a fim de identificar as possíveis orientações para o cuidado no pós-operatório de ortopedia.

A segunda etapa diz respeito a escolha do tipo de software que seria desenvolvido e da realização de uma pesquisa bibliográfica para maior aprofundamento acerca da temática. O levantamento aconteceu em bancos de dados científicos, como Scientific Electronic Library Online (SciELO) e Medical Literature Analysis and Retrieval System Online (MEDLINE). A partir disso, adquriu-se maior entendimento sobre os principais cuidados no pós-operatório de ortopedia pediátrica.

A terceira etapa consistiu no desenvolvimento do aplicativo, para isto, contratou-se os serviços de uma empresa especializada na área de desenvolvimento de softwares. Posteriormente, foi apresentado o perfil epidemiológico traçado na primeira etapa e os dados coletados na segunda etapa. Com isso, a empresa iniciou o processo de construção do aplicativo, a saber: configuração, especificações de design visual e funcionalidades. É importante salientar que todo o processo foi acompanhado pelos pesquisadores, por meio de reuniões mensais.

Após a etapa de desenvolvimento, o estudo foi submetido a quarta etapa, a qual consistiu na realização vários testes pelos pesquisadores e programadores, utilizando o aplicativo TestFlight do Sistema IOS, sendo a última versão 1.1.0 registrado no Instituto Nacional de Propriedade Industrial sob número do processo: 512020002559-5.

Por fim, a quinta etapa diz respeito ao processo de avaliação e validação de conteúdo pelos juízes. Participaram desta etapa 8 enfermeiros e 11 mães. Sobre os critérios de inclusão dos enfermeiros: atuar na clínica pediátrica e ter tido contato com criança de pós-operatório de cirúrgica ortopédica durante o período de validação. Não foram considerados aptos os enfermeiros residentes, em cargo de chefia e com tempo de formação menor que 2 anos. Sobre os critérios de inclusão da mãe: ter filho na faixa etária 01 a 12 anos de idade submetido à cirúrgica ortopédica no hospital em questão.

Após a leitura e assinatura do TCLE, os juízes eram apresentados ao aplicativo, momento em que o manuseavam e verificavam suas funcionalidades. Posteriormente, após o tempo necessário para o devido manuseio do aplicativo, Ihes eram entregue o instrumento de avaliação. Foram desenvolvidos dois questionários para os grupos de participantes (mãe e enfermeiro) adaptado de Pinheiro AB, et al. (2018). A ferramenta foi composta por 12 perguntas, divididas em cinco blocos. O bloco I relacionado aos dados de identificação dos participantes e os blocos II, III, IV e V foi composto por perguntas específicas (conforme ilustra o Quadro 1.

Destaca-se, que ambos possuem os mesmos itens de avaliação, e que somente no questionário das integrantes (mães) foram realizadas adequações na linguagem do conteúdo para melhor compreensão das participantes. Para verificar o grau de concordância ou discordância dos participantes, optou-se pela escala de resposta psicométrica de Likert, ou seja, cada pergunta dos blocos II, III, IV e V possuíam cinco alternativas (discordo totalmente, discordo parcialmente, indiferente, concordo parcialmente e concordo totalmente). 
Posteriormente, as informações extraídas dos questionários, foram digitados e agrupados diretamente em uma planilha do programa Microsoft Excel $2016^{\circledR}$, em seguida foram codificados em forma de gráficos e tabelas por intermédio do software IBM SPSS ${ }^{\circledR}$ versão 22.0. Ademais, os testes utilizados para análise das variáveis foram: o Índice de Validade de Conteúdo (IVC) aplicado para mensurar o grau de concordância e o Coeficiente Alfa de Cronbach para medir o grau de confiabilidade.

Quadro 1 - Parâmetros avaliados por enfermeiros e mães.

\begin{tabular}{|c|c|}
\hline Itens de Assertivas - Enfermeiros & Itens de Assertivas - Mães \\
\hline $\begin{array}{l}\text { Bloco I - Identificação: Pseudônimo, Gênero, } \\
\text { Idade e tempo de formação }\end{array}$ & $\begin{array}{l}\text { Bloco I - Identificação: Pseudônimo, Idade da } \\
\text { mãe, idade da criança e local do trauma }\end{array}$ \\
\hline $\begin{array}{l}\text { Bloco Il- Avaliação do funcionamento } \\
\text { 1. O aplicativo é de fácil utilização pelo usuário; } \\
\text { 2. O aplicativo é de fácil aprendizagem e pode ser } \\
\text { usado por qualquer usuário que tenha } \\
\text { habilidade para leitura e interpretação de textos; } \\
\text { 3. O aplicativo é de fácil instalação e pode ser } \\
\text { acessado em qualquer lugar e hora pelo } \\
\text { usuário. }\end{array}$ & $\begin{array}{l}\text { Bloco Il - Avaliação do funcionamento } \\
\text { 1. É fácil de usar; } \\
\text { 2. É fácil de aprender. Pode ser usado por } \\
\text { qualquer mãe que saiba ler e interpretar os } \\
\text { textos e imagens; } \\
\text { 3. Fácil de baixar no celular e pode usar em } \\
\text { qualquer lugar e hora. }\end{array}$ \\
\hline $\begin{array}{l}\text { Bloco III - Avaliação da interação do usuário } \\
\text { 4. Os ícones, figuras e imagens da tela do } \\
\text { aplicativo estão organizados de forma simples } \\
\text { que permite o fácil uso pelo usuário; } \\
\text { 5. Os recursos disponibilizados na tela do } \\
\text { aplicativo (texto, ícone, imagens) são fáceis de } \\
\text { leitura e compreensão pelo usuário. }\end{array}$ & $\begin{array}{l}\text { Bloco III - Avaliação da interação do usuário } \\
\text { 4. Os desenhos estão organizados de forma } \\
\text { simples que permite o fácil uso; } \\
\text { 5. Os textos e os desenhos são fáceis de ler e } \\
\text { entender. }\end{array}$ \\
\hline $\begin{array}{l}\text { Bloco IV - Avaliação da rapidez do aplicativo } \\
\text { 6. O tempo para realizar as tarefas no aplicativo } \\
\text { não é demorado; } \\
\text { 7. O aplicativo ajuda na prática profissional no } \\
\text { cuidado com a criança de pós-operatório de } \\
\text { ortopedia. }\end{array}$ & $\begin{array}{l}\text { da rapidez do aplicativo } \\
\text { ver os desenhos e os textos; } \\
\text { er o cuidado com a criança } \\
\text { a. }\end{array}$ \\
\hline $\begin{array}{l}\text { Bloco V - Avaliação da qualidade do aplicativo } \\
\text { 8. O aplicativo possui uma linguagem clara e } \\
\text { objetiva que é de fácil entendimento pelo } \\
\text { usuário; } \\
\text { 9. O aplicativo aborda os principais cuidados no } \\
\text { pós-operatório de cirurgia ortopédica. } \\
\text { 10. Este aplicativo garante segurança e sigilo das } \\
\text { informações do usuário cadastrado, pois para } \\
\text { usar foi necessário o uso de senha. } \\
\text { 11. Ao usar o aplicativo você se sente satisfeito (a) } \\
\text { com o tipo de apresentação e abordagem dos } \\
\text { cuidados no pós-operatório de cirurgia } \\
\text { ortopédica. }\end{array}$ & $\begin{array}{l}\text { Bloco V - Avaliação da qualidade do aplicativo } \\
\text { 8. Usa textos e desenhos que são fáceis de } \\
\text { entender; } \\
\text { 9. Foi útil para entender os tipos de cuidados com } \\
\text { a criança depois da cirurgia; } \\
\text { 10. O aplicativo garante segurança e segredo das } \\
\text { informações, pois para usar é necessário o uso } \\
\text { de senha; } \\
\text { 11. Ao usar o aplicativo você se sente satisfeita com } \\
\text { o tipo de textos e desenhos apresentados. }\end{array}$ \\
\hline
\end{tabular}

Fonte: Cantão BCG, et al., 2021.

Para avaliar a concordância dos participantes, foi empregado o IVC, que é uma medida quantitativa bastante utilizada na área da saúde e no uso da escala de Likert, serve para medir o grau de concordância dos participantes. Para o conteúdo ser considerado validado, o IVC Geral deve possuir índice de concordância maior que 0,80 (ALEXANDRE NMC e COLUCI MZO, 2011; SOUZA AC, et al., 2017)

No que se refere a mensuração do grau de confiabilidade, optou-se pelo índice de Alfa de Cronbach, em razão de ser bastante utilizado em pesquisa envolvendo escala de Likert. É aplicado quando se pretende avaliar o grau de confiabilidade e a consistência interna (homogeneidade) dos itens presente em um instrumento. O parâmetro mínimo aceitável é o alfa 0,70 (SOUZA AC, et al., 2017). 


\section{RESULTADOS}

O aplicativo "PED-ORTO" foi projetado em versão híbrida para funcionar gratuitamente em dispositivos móveis smartphone ou tablete nas plataformas Android ou IOS. Para mais, os resultados da pesquisa foram divididos em três tópicos: desenvolvimento do aplicativo, perfil dos participantes e avaliação do funcionamento do aplicativo.

\section{Desenvolvimento do aplicativo}

O aplicativo foi desenvolvido para permitir o compartilhamento de informações entre o enfermeiro e a cuidadora (mãe), e apresenta uma sequência de ações que se inicia após o cadastro e login do usuário. No caso dos enfermeiros, após o login, o aplicativo permite o acesso a lista de mães cadastradas e apresentação dos cuidados para o ambiente hospitalar e doméstico, que abrangem as funções de respiração, circulação, neurológica, eliminação, segurança, conforto e orientações sobre o tipo, horário e dosagem das medicações prescritas pelo médico. Além disso, permite ao enfermeiro selecionar os tipos de cuidados (imagem e texto) que melhor se adequem com a intervenção cirúrgica do paciente com objetivo de auxiliar as mães nos cuidados a serem realizados no ambiente hospitalar e no domicílio após alta médica (Figura 1).

Figura 1 - Telas do aplicativo.
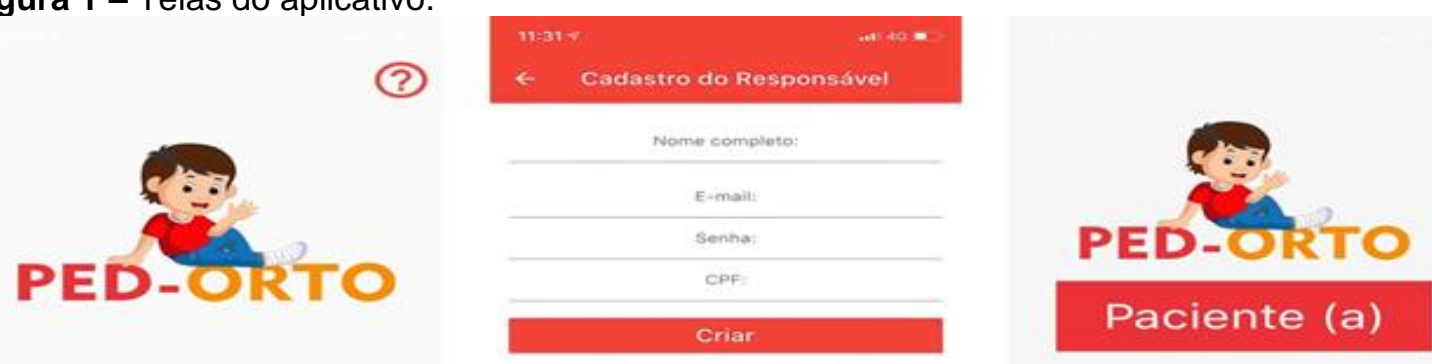

\section{Paciente (a)}

\section{Paciente}

\section{Enfermeiro(a)}

A

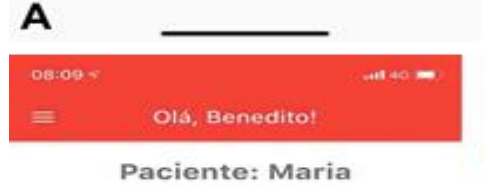

\section{A Ambiente Doméstico}

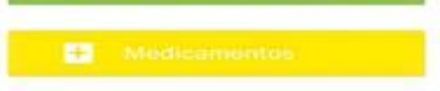

$\$$ Visualizar Cuidados

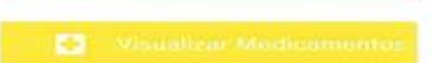

+ Dar alta dos cuidados

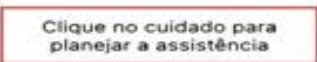

D
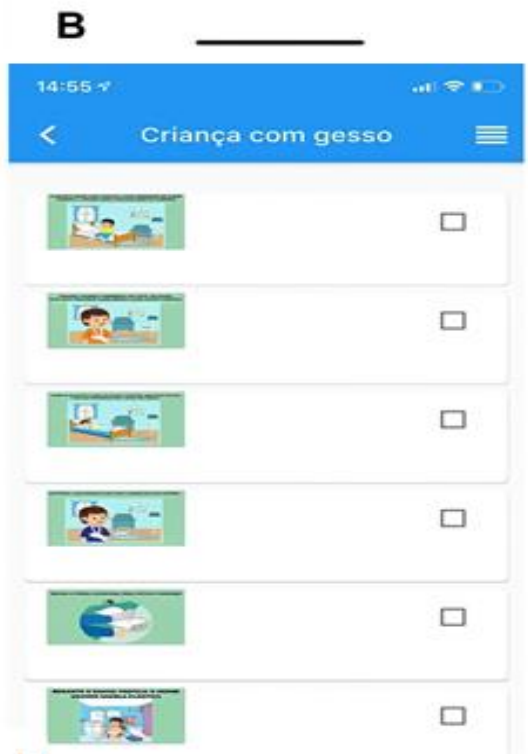

E

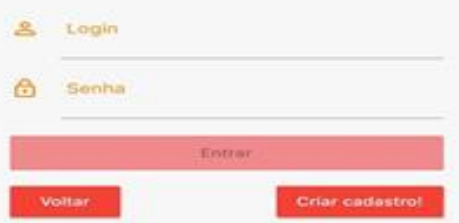

C
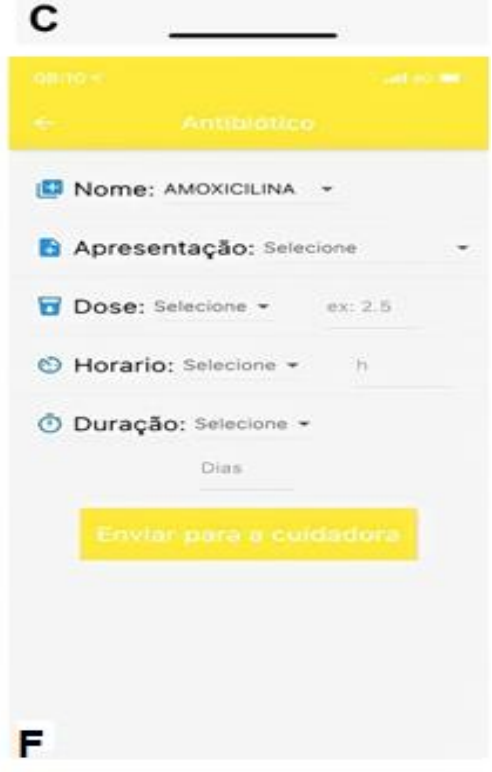

Legenda: A - Tela inicial; B - Cadastro do usuário; C - Login do usuário; D - Cuidados no ambiente doméstico; E - Lista de cuidados para criança com gesso; F - Tela para introdução dos medicamentos. Fonte: Cantão BCG, et al., 2021. 
Ademais, para as mães, a visualização do conteúdo e das mensagens automáticas (horário e dosagem da mediação) é realizada por meio de textos e imagens que podem ser acessadas em dois momentos, 0 primeiro é no ambiente hospitalar e o segundo no ambiente doméstico. A apresentação consiste em uma sequência de cuidados previamente selecionados pelo enfermeiro que ficará disponível para as mães durante todo tratamento, em média de 20 a 30 dias, após esse período é dado alta no aplicativo pelo enfermeiro, não permitindo a exibição ou introdução de novos cuidados.

Vale ressaltar que o acesso é individualizado e seguro por meio do uso de senha, permitindo ao usuário a ampliação das imagens por meio do zoom e acesso ao enfermeiro pelo chat do aplicativo. Ademais, no caso dos cuidados para o domicílio, o mesmo dá autonomia para as mães aceitarem ou não receber mensagens de alerta sobre a dosagem e horário dos medicamentos.

\section{Perfil dos participantes}

No caso dos enfermeiros, constatou-se que $100 \%$ dos participantes eram do sexo feminino com a maioria pertencente a faixa etária acima de 40 anos e tempo de formação superior a 10 anos. Em relação as mães, a concentração ficou na faixa etária abaixo dos 30 anos e a dos filhos, a maior proporção ficou em torno de 05 a 10 anos de idade. Sobre o sexo da criança, o masculino foi predominante. em torno de $81,8 \%$. Em relação ao local do trauma (fratura) do infante, a maioria ocorreu nos membros superiores com $72,7 \%$ dos casos registrados acompanhado dos membros inferiores com $27,3 \%$ (Tabela 1).

Tabela 1 - Perfil dos dos participantes da pesquisa.

\begin{tabular}{|c|c|c|c|}
\hline \multicolumn{4}{|c|}{ Perfil dos Enfermeiros } \\
\hline \multicolumn{2}{|c|}{ Variáveis } & Frequência & $\%(n=8)$ \\
\hline \multirow{2}{*}{ Sexo } & Masculino & - & - \\
\hline & Feminino & 8 & $100 \%$ \\
\hline \multirow{3}{*}{ Faixa etária (anos) } & $\leq 40$ & 2 & $25 \%$ \\
\hline & $41-49$ & 3 & $37,5 \%$ \\
\hline & $\geq 50$ & 3 & $37,5 \%$ \\
\hline \multirow{4}{*}{$\begin{array}{l}\text { Tempo de formação } \\
\text { (anos) }\end{array}$} & $\leq 10$ & 2 & $25 \%$ \\
\hline & $11-20$ & 3 & $37,5 \%$ \\
\hline & $21-30$ & 2 & $25,5 \%$ \\
\hline & $>30$ & 1 & $12,5 \%$ \\
\hline \multicolumn{4}{|c|}{ Perfil das Mães } \\
\hline & Variáveis & Frequência & $\%(n=11)$ \\
\hline \multirow{3}{*}{$\begin{array}{l}\text { Faixa etária da mãe } \\
\text { (anos) }\end{array}$} & $\leq 30$ & 8 & $72,7 \%$ \\
\hline & $31-49$ & 3 & $27,3 \%$ \\
\hline & $\geq 50$ & - & - \\
\hline \multirow{2}{*}{ Sexo da criança } & Masculino & 9 & $81,8 \%$ \\
\hline & Feminino & 2 & $18,2 \%$ \\
\hline \multirow{3}{*}{$\begin{array}{l}\text { Faixa etária da criança } \\
\text { (anos) }\end{array}$} & $<5$ & 1 & $9,1 \%$ \\
\hline & $5-10$ & 7 & $63,6 \%$ \\
\hline & $\geq 10$ & 3 & $27,3 \%$ \\
\hline \multirow{3}{*}{ Localização do trauma } & Membros superiores & 8 & $72,7 \%$ \\
\hline & Membros inferiores & 3 & $27,3 \%$ \\
\hline & Outros locais & - & - \\
\hline
\end{tabular}

Fonte: Cantão BCG, et al., 2021.

\section{Avaliação do funcionamento do aplicativo}

O aplicativo "PED-ORTO" obteve um IVC Geral de 0,85 , na categoria "enfermeiros", considerado bom no quesito de concordância entre os participantes. Quando analisado separadamente, a maioria dos itens apresentaram uma pontuação superior a 0,80. Entretanto, os itens 2 e 3 que tratam respectivamente da aprendizagem e instalação do aplicativo ficaram abaixo do índice recomendado. Vale ressaltar, que essa variação não interfere no uso do aplicativo, visto que o índice geral ficou acima da média recomendada. No 
grupo das mães, o índice apresentou um escore maior em comparação com o grupo de enfermeiros, ficando com o IVC Geral de 0,89, sendo que neste grupo os itens 6 , o item 7 e o item 9 apresentaram pontuação máxima. Já o item 3, assim como no grupo anterior ficou abaixo do índice recomendado (Tabela 2).

Ao convergir os dados dos enfermeiros e das mães, obteve-se um Índice de Validade de Conteúdo Geral de 0,88 , resultado considerado positivo para validação do aplicativo. Vale salientar, que o item 6 apresentou pontuação máxima, com um ótimo desempenho na avaliação dos dois grupos. O estudo teve apenas o item $3 \mathrm{com}$ escore abaixo do índice recomendado. Tal intepretação, reforça que a maior dificuldade, encontra-se na instalação do aplicativo 'PED-ORTO” pelos usuários. Felizmente, essa variação não interfere no uso do aplicativo, visto que o IVC Geral do estudo ficou acima da média recomendada para validação.

Tabela 2 - Avaliação por critérios e IVC pelas participantes.

\begin{tabular}{|c|c|c|c|}
\hline \multicolumn{4}{|c|}{ Avaliação dos enfermeiros } \\
\hline Critérios de avaliação & Assertivas & \% Aprovação & $\mathrm{IVC}^{*}$ \\
\hline \multirow{3}{*}{ Funcionamento } & Item 1 & $87.5 \%$ & 0.88 \\
\hline & Item 2 & $82.5 \%$ & 0.75 \\
\hline & Item 3 & $62.5 \%$ & 0.63 \\
\hline \multirow{2}{*}{ Interação do usuário } & Item 4 & $82.5 \%$ & 0.88 \\
\hline & Item 5 & $85.0 \%$ & 0.88 \\
\hline \multirow{2}{*}{ Rapidez } & Item 6 & $90.0 \%$ & 1.00 \\
\hline & Item 7 & $85.0 \%$ & 0.88 \\
\hline \multirow{4}{*}{ Qualidade } & Item 8 & $87.5 \%$ & 0.88 \\
\hline & Item 9 & $85.0 \%$ & 0.88 \\
\hline & Item 10 & $87.5 \%$ & 0.88 \\
\hline & Item 11 & $87.5 \%$ & 0.88 \\
\hline \multicolumn{2}{|c|}{ Avaliação Geral } & $83.9 \%$ & 0.85 \\
\hline \multicolumn{4}{|c|}{ Avaliação das mães } \\
\hline Critérios de avaliação & Assertivas & \% Aprovação & IVC $^{*}$ \\
\hline \multirow{3}{*}{ Funcionamento } & Item 1 & $87.3 \%$ & 0.91 \\
\hline & Item 2 & $89.1 \%$ & 0.91 \\
\hline & Item 3 & $67.3 \%$ & 0.55 \\
\hline \multirow{2}{*}{ Interação do usuário } & Item 4 & $90.9 \%$ & 0.91 \\
\hline & Item 5 & $90.9 \%$ & 0.91 \\
\hline \multirow{2}{*}{ Rapidez } & Item 6 & $92.7 \%$ & 1.00 \\
\hline & Item 7 & $96.4 \%$ & 1.00 \\
\hline \multirow{4}{*}{ Qualidade } & Item 8 & $85.5 \%$ & 0.82 \\
\hline & Item 9 & $89.1 \%$ & 1.00 \\
\hline & Item 10 & $89.1 \%$ & 0.91 \\
\hline & Item 11 & $85.5 \%$ & 0.91 \\
\hline \multicolumn{2}{|c|}{ Avaliação Geral } & $87.6 \%$ & 0.89 \\
\hline \multicolumn{4}{|c|}{ Avaliação geral dos participantes (enfermeiros e mães) } \\
\hline Critérios de avaliação & Assertivas & \% Aprovação & $\mathrm{IVC}^{*}$ \\
\hline \multirow{3}{*}{ Funcionamento } & Item 1 & $87.4 \%$ & 0.89 \\
\hline & Item 2 & $86.3 \%$ & 0.87 \\
\hline & Item 3 & $65.3 \%$ & 0.58 \\
\hline \multirow{2}{*}{ Interação do usuário } & Item 4 & $87.4 \%$ & 0.89 \\
\hline & Item 5 & $88.4 \%$ & 0.89 \\
\hline \multirow{2}{*}{ Rapidez } & Item 6 & $91.6 \%$ & 1.00 \\
\hline & Item 7 & $91.6 \%$ & 0.95 \\
\hline \multirow{4}{*}{ Qualidade } & Item 8 & $86.3 \%$ & 0.84 \\
\hline & Item 9 & $87.4 \%$ & 0.95 \\
\hline & Item 10 & $88.4 \%$ & 0.89 \\
\hline & Item 11 & $86.3 \%$ & 0.89 \\
\hline \multicolumn{2}{|c|}{ Avaliação Geral } & $86.0 \%$ & 0.88 \\
\hline
\end{tabular}

Fonte: Cantão BCG, et al., 2021. 
$\mathrm{Na}$ avaliação da confiabilidade do questionário, levou-se em consideração os valores do alfa de Cronbach dos dois grupos de participantes. A média não apresentou diferença estatística significante quando comparados entre si, cujo os valores oscilaram de 0.894 a 0.895 obtendo no final a Média Geral de 0.906 , resultado considerado "Alta Confiabilidade", em uma escore que varia de 0 a 1. Portanto, é possível concluir que os itens são homogêneos e que tendem a se agrupar dentro escala (Tabela 3).

Ao correlacionar os dados obtidos por meio das respostas dos 19 participantes, atestou-se que ambos os índices usados para validação do aplicativo "PED-ORTO", ficaram com taxa de aprovação maior que $86 \%$ e os escores apresentaram respostas positivas para uso do aplicativo no âmbito dos cuidados para crianças de pós-operatório de ortopedia.

Tabela 3 - Resultados finais da avaliação do aplicativo, pelos participantes.

\begin{tabular}{cccc}
\hline Validação do aplicativo & \% Aprovação & IVC $^{*}$ & Alfa Cronbach \\
\hline Avaliação das Mães & $87.6 \%$ & 0.89 & 0.895 \\
\hline Avaliação dos Enfermeiros & $83.9 \%$ & 0.85 & 0.894 \\
\hline Avaliação Geral & $\mathbf{8 6 . 0 \%}$ & $\mathbf{0 . 8 8}$ & $\mathbf{0 . 9 0 6}$ \\
\hline
\end{tabular}

Fonte: Cantão BCG, et al., 2021.

\section{DISCUSSÃO}

No que concerne ao emprego da tecnologia aplicada a saúde, aplicativos móveis são uma estratégia que vêm sendo utilizadas com mais frequência para melhorar a comunicação entre o cliente e o profissional, pois tais, representam uma alternativa viável devido ao grande potencial de alcance. $O$ aplicativo tem como finalidade orientar e incentivar mudança do comportamento humano, fornecendo direção e propósitos que permite uma melhor facilidade no acesso à informação e no gerenciamento do cuidar (ROCHA FS, et al., 2017).

Para a enfermagem, essa modernização da assistência melhora o processo de tomada de decisão clínica e a qualidade da prestação de cuidados. Além disso, estima-se que até 2025, a temática de tecnologias seja considerada como componente obrigatório da grade curricular do curso (ALVES AG, et al., 2020). Assim sendo, o enfermeiro deve possuir disponibilidade para aprender as novas técnicas, para que possa compreender o seu funcionamento e dessa forma consiga adaptar-se ao uso da tecnologia em seu ambiente de trabalho (ARAÚJO MAN, et al., 2017; PERISSÉ L, et al., 2019).

Considerando o trauma na infância um acontecimento frequente, na literatura pesquisada não foi identificado um aplicativo para dispositivos móveis projetado com imagens e textos direcionado para orientar as mães quando ao manejo correto no pós-operatório de ortopedia.

Diversos estudos têm demonstrado, que as lesões decorrentes de trauma de acidente e violência, constituem um problema para os serviços de saúde, devido representar importante causa de morte ou hospitalização, sendo considerado mais incidente na de 01 a 49 de idade (BRASIL, 2019).

Além disso, as lesões traumáticas decorrentes de queda são consideradas o tipo de acidente mais frequentes, sendo mais comum nos membros superiores, com uma ocorrência maior no sexo masculino e no início da adolescência (FIORIN BH, et al., 2020).

Na pesquisa de Cantão BCG, et al. (2020), tais dados se repetem, uma vez que o resultado mostra que a parte do organismo mais afetada pelas lesões traumáticas foram os membros superiores $(72,7 \%)$ seguido dos membros inferiores $(27,3 \%)$. Essa incidência aumenta consideravelmente quando levado em consideração o sexo e a idade, sendo maior nos meninos $(81,8 \%)$ e na faixa etária de até 10 anos de idade (72,7\%).

Vale ressaltar, que as causas dos traumas na infância são decorrentes de um conjunto de fatores que combinados entre si, intensificam a probabilidade de acidentes e lesões, dos quais, a idade e o sexo são uma das principais variáveis, pois esta condiz com as etapas do desenvolvimento psicomotor que reflete na atividade diária da criança. Em um estudo realizado no serviço de urgência, em Campinas, Estado de São 
Paulo foi observado que as lesões eram mais frequentes no sexo masculino com $63,3 \%$ dos atendimentos com intervalo de idade correspondente de 06 a 09 anos (BEM MAM, et al., 2008; FIORIN BH, et al., 2020).

No que se refere aos parâmetros utilizados para validação do aplicativo, optou-se pelos índices de IVC e o alfa de Cronbach. É importante frisar que para avaliar o grau de usabilidade, deve se levar em consideração as variáveis e as especificidade de cada usuário, pois são considerados fatores determinantes para se avaliar eficácia, eficiência e satisfação de um produto (PAZ FJ e LEITE MCD, 2017).

A avaliação da usabilidade do aplicativo levou em consideração os critérios de funcionamento, rapidez, interação do usuário e qualidade como propósito de analisar o grau de concordância e identificar possíveis falhas na programação do software. Sabe-se que avaliação da usabilidade é uma estratégia de validação que vem sendo cada vez mais utilizada quando se trata de construção de softwares, principalmente quando se pretende avaliar os conceitos pedagógico, de design e execução de tarefas dos usuários (VERAS WTA, et al., 2020).

Em relação a concordância, no geral, pode-se afirmar que o índice mede as características necessárias para a validação do aplicativo, pois os dados analisados das respostas dos enfermeiros e das mães foram considerados uniformes com IVC Geral acima de 0,80. Vale destacar que, para estudo envolvendo mais de seis avaliadores, o índice dos itens calculados deve apresentar concordância igual ou superior 0,80 (ALEXANDRE NMC e COLUCI MZO, 2011; COSTA CC, et al., 2020).

É relevante frisar que apesar do resultado ser favorável para o uso da aplicativo, a pesquisa demostra que ele necessita de atualização para correção do item 3 que corresponde a instalação do aplicativo. A baixa escore, pode ser explicada pelo fato do aplicativo durante a fase de validação não se encontrar disponível para download nas plataformas IOS e Android. O software foi disponibilizado aos participantes por meio link, o que nos leva acreditar que tal acontecimento possa ter comprometido a avaliação deste componente.

No geral, pode-se afirmar que o índice mede as características necessárias para a validação do aplicativo, pois o IVC Geral ficou acima da média recomendada, e que apesar do resultado ser positivo, a pesquisa demostra que o aplicativo necessita de atualização para correção do item 3. Reforça-se que a equipe de programadores foi acionada, para que as devidas correções fossem realizadas. As atualizações dos sistemas operacionais são procedimentos comuns na área de tecnologia e servem para corrigir falhas internas identificadas pelo fabricante ou introduzir novas funções (GAZOLA R, 2020).

$\mathrm{Na}$ análise da confiabilidade do instrumento de validação do aplicativo, obteve um Alto desempenho com alfa maior que 0,88 nos dois grupos, e média geral em torno de 0,906 , demostrando que a escala apresenta uma alta convergência interna e homogeneidade dos itens. Seguindo a classificação de Gottems LBD, et al., (2018), um escore maior que 0,90 é considerado muito alto, sendo, portanto, excelente.

Em síntese, o aplicativo, atingiu sua finalidade ao permitir a interação entre o enfermeiro e a mãe. Os resultados demonstram uma boa aceitação conforme Tabela 6, pois no item 11 que trata sobre satisfação do usuário, o estudo apresentou IVC Geral de 0,89 e taxa de aprovação de 86,3\%. Quando averiguado isoladamente, 0 item 11 obteve maior taxa de aprovação entre as mães.

Além disso, acredita-se que o aplicativo possa servir como subsídios aos enfermeiros que atuam na área de ortopedia para o planejamento da assistência de enfermagem e desta forma possa auxiliar e instigar as mães nos cuidados da criança durante o período de pós-operatório imediato e mediato. Como limitação deste estudo, tiveram-se o tamanho amostral em decorrência da pandemia da COVID-19, com consequente redução no número de cirurgias durante o período de validação. Sendo indicado como sugestão para trabalhos futuros, a ampliação dos locais de estudo e por uma quantidade maior de participantes.

\section{CONCLUSÃO}

O aplicativo "PED-ORTO" é um produto que pode ser utilizado no âmbito hospitalar que tenha como finalidade auxiliar as mães nos cuidados de pós-operatório de ortopedia pediátrica, visto que os resultados de validação referentes a usabilidade demostraram um alto grau de concordância e confiabilidade. Além disso, 
o aplicativo tem aplicabilidade imediata, uma vez que estará disponível para os profissionais de saúde e cuidadores de criança que serão submetidas a cirurgia ortopédica, bem como permite a inserção social, ao instigar a cooperação das mães no manejo da criança de pós-operatório de ortopedia.

\section{REFERÊNCIAS}

1. ALEXANDRE NMC, COLUCI MZO. Validade de conteúdo nos processos de construção e adaptação de instrumentos de medidas. Ciênc. saúde coletiva, 2011; 16(7): 3061-68.

2. ALVES AG, et al. Information and communication technology in nursing education. Acta Paul Enferm. 2020; 33: 1-8.

3. ARAÚJO MAN, et al. Perfil sociodemográfico dos enfermeiros da rede hospitalar. Rev. enfermagem UFPE. 2017; 11(11): 4716-25.

4. BARCELOS RS, et al. Acidentes por quedas, cortes e queimaduras em crianças de 0-4 anos: coorte de nascimentos de Pelotas, Rio Grande do Sul, Brasil, 2004. Cadernos de Saúde Pública, 2017; 1(33): e00139115.

5. BEM MAM, et al. Epidemiologia dos pequenos traumas em crianças atendidas no Hospital Infantil Joana de Gusmão. Arquivos Catarinenses de Medicina, 2008; 37(2): 59-6.

6. BRASIL. Departamento de Vigilância de Doenças e Agravos Não Transmissíveis e Promoção da Saúde: Instrutiva notificação de violência interpessoal e autoprovocada. Ministério da Saúde; 2019. Disponível em: https://antigo.saude.gov.br/images/pdf/2019/dezembro/05/viva-inquerito-2017.pdf. Acessado em: 27 de março de 2021.

7. BUTCHER HK, et al. Nursing interventions classification (NIC). Elsevier Health Science, 2018.

8. CANTÃO BCG, et al. Perfil epidemiológico de traumas ortopédicos pediátricos em um hospital do interior do Pará. REAS, 2021; 13(2): e6265.

9. COSTA CC, et al. Construção e validação de uma tecnologia educacional para prevenção da sífilis congênita. Acta Paul Enferm, 2020; 33:1-8.

10. CUNHA MLR, et al. aplicativo para preparar criança / família para punção venosa: relato de experiência. Rev. Brasileira de Enfermagem, 2018; 71(3): 1474-78.

11. FILÓCOMO FRF, et al. Perfil dos acidentes na infância e adolescência atendidos em um hospital público. Acta Paulista de Enfermagem, 2017; (3): 287-294.

12. FIORIN BH, et al. Validade e confiabilidade do questionário de avaliação multidimensional após o infarto do miocárdio. Rev. Eletr. Enferm. 2020. 1-8.

13. GAZOLA R. In: ADDEE+. Por que manter meus softwares atualizados. 2020. Disponível em: https://addee.com.br/blog/softwares-atualizados. Acessado em: 27 de março de 2021.

14. GOTTEMS LBD, et al. Boas práticas no parto normal: análise da confiabilidade de um instrumento pelo Alfa de Cronbach. Rev. Latino-Am. Enfermagem, 2018: 1-8.

15. HERDMAN T. Diagnósticos de Enfermagem da NANDA Internacional. Diagnósticos de Enfermagem da NANDA Definições e classificação. Ed. 2017. 2015. 127-442.

16. IBGE. In: Instituto Brasileiro de Geografia e Estatística. PNAD Contínua TIC 2018: Internet chega a 79,1\% dos domicílios do país. 2020. Disponível em: https://agenciadenoticias.ibge.gov.br/agencia-sala-de-imprensa/2013agencia-de-noticias/releases/27515-pnad-continua-tic-2018-internet-chega-a-79-1-dos-domicilios-do-pais. Acessado em: 27 de março de 2021.

17. KRUK ME, et al. High-quality health systems in the Sustainable Development Goals era: time for a revolution. The Lancet, 2018; 6(1): 1196-52.

18. PAZ FJ, LEITE MCD. In: Jornada da Pós-Graduação e Pesquisa, Avaliação da usabilidade do aplicativo comuniquese: um app para pacientes que sofreram AVC. Revista da Jornada de Pós-Graduação e Pesquisa-Congrega Urcamp, 2017: 292-308.

19. PERISSÉ L, et al. Desafios e limitações do enfermeiro inerentes à incorporação de novas tecnologias. Rev. Enfermagem Atual. 2019; 87(25): 87-89.

20. PINHEIRO AB, et al. Uso do aplicativo leite solidário para gerenciamento de doação de leite materno. In: Defesa Final do Mestrado Cirurgia e Pesquisa Experimental - CIPE, 2018, Belém.

21. ROCHA FS, et al. In: Seminário de Tecnologias Aplicadas em Educação e Saúde (STAES). Uso de apps para a promoção dos cuidados à saúde., $2017 . \quad$ Disponível em: https://www.revistas.uneb.br/index.php/staes/article/view/3832. Acessado em: 27 de março de 2021.

22. SILVA JP, GARANHANI ML. O significado do cuidado perioperatório para a criança cirúrgica. Rev. Eletrônica de Enfermagem, 2011; 13(2): 259-68.

23. SOUZA AC, et al. Propriedades psicométricas na avaliação de instrumentos: avaliação da confiabilidade e da validade. Epidemiol. Serv. Saúde, 2017; 26(3): 649-59.

24. VERAS WTA, et al. Desenvolvimento e Avaliação da Usabilidade em Aplicativo Desktop para o Ensino da Programação Orientada a Objetos. In: V Congresso sobre Tecnologia na Educação, 2020, João Pessoa. 